\title{
Evaluation of the epicardial fat thickness in patients with the diagnosis of cardiac syndrome $\mathrm{X}$
}

\author{
Macit Kalçık ${ }^{1 *}$, Mahmut Yesin ${ }^{2}$, Ahmet Güner ${ }^{3}$ and Emrah Bayam ${ }^{4}$ \\ ${ }^{1}$ Department of Cardiology, Hitit University Faculty of Medicine, Çorum, Turkey \\ ${ }^{2}$ Department of Cardiology, Kars Harakani State Hospital, Kars, Turkey \\ ${ }^{3}$ Department of Cardiology, Kosuyolu Kartal Training and Research Hopital, Istanbul, Turkey \\ ${ }^{4}$ Department of Cardiology, Umraniye Training and Research Hospital, Istanbul, Turkey
}

\begin{abstract}
Introduction: Cardiac syndrome X (CSX), also named as microvascular angina, is defined as effort angina with detectable ischemia on noninvasive tests without any evidence of stenosis or vasospasm of epicardial coronary arteries during invasive coronary angiography. Impaired coronary microcirculation, inflammation, and insulin resistance resulting in endothelial dysfunction are accepted etiological factors for CSX. Recently increased epicardial fat tissue (EFT) thickness has been associated with hypertension, insulin resistance, metabolic syndrome, and coronary artery disease in general population. In this study, we aimed to evaluate EFT values in patients with CSX.

Methods: This study enrolled 145 patients (mean age: $54.3 \pm 8.2$, male: 59 ) who diagnosed with CSX and 150 healthy controls (mean age: $55.9 \pm 7.3$, male: 66 ) between May 2016 and January 2018. All patients underwent transthoracic echocardiography and EFT thickness was measured from a parasternal long-axis view as the hypoechoic space on the right ventricular free wall. All demographic, laboratory and echocardiographic parameters were recorded into a dataset and compared between CSX patients and controls.

Results: There was no significant difference in terms of demographic, laboratory and echocardiographic parameters between CSX patients and the controls. The mean EFT thickness was significantly higher in CSX patients than the controls (5.6 $\pm 1.1 \mathrm{vs.} 4.9 \pm 0.8 \mathrm{~mm} ; \mathrm{p}<0.001)$. Multiple logistic regression analysis showed that increased EFT thickness was an independent predictor of CSX (Odds ratio: 1.289; CI\%95: 0.219-0.946; $\mathrm{p}=0.021$ ). In receiver operating characteristic curve analyses, EFT thickness above $5.3 \mathrm{~mm}$ predicted CSX with a sentivity of $69 \%$ and a specificity of $64 \%$ (AUC: $0.721 ; 95 \%$ CI: 0.661 to 0.781 ; p<0.001).
\end{abstract}

Conclusion: EFT thickness values were observed significantly higher in CSX patients as compared to healthy controls. Increased EFT thickness may be associated with mechanisms that play role in the pathogenesis of CSX.

\section{Introduction}

A significant proportion (20-30\%) of patients presenting with typical angina pectoris have angiographically normal coronary arteries [1]. Although noncardiac causes may be responsible for the chest-pain, a considerable subset of these patients have true angina due to myocardial ischaemia in the absence of angiographically significant coronary stenosis [2] The term cardiac syndrome X (CSX) are used to describe patients with typical angina pectoris and a positive stress test (classic down-sloping ST segment depression on treadmill exercise test, and/ or a reversible perfusion defect on radionuclear myocardial perfusion scan), in the absence of significant coronary stenosis on angiography and other cardiac diseases [3]. Coronary microvascular dysfunction was suggested to be the underlying pathophysiologic mechanism. Moreover, endothelial dysfunction with subsequent microvascular ischemia has been implicated as an important contributing factor [4]. While, several studies reported that patients with CSX supposed to have excellent long-term clinical outcome [5] other clinical reports have been reported that a considerable percentage of those patients might have a higher risk of unfavorable clinical outcomes [6].

Epicardial fat tissue (EFT) is a true visceral adipose tissue, deposited in proximity to the atrium, the right ventricle's free wall, and left ventricular apex of the heart [7]. Under normal conditions EFT has important functions as protecting vascular functions and providing energy requirement of the heart by producing free fatty acids which are energy sources for myocardium. However, when volume of the epicardial adipose tissue increases, this tissue acquires a prothrombotic and proinflammatory characteristics [8]. Increased EFT thickness has been defined as a cardiometabolic risk factor with a possible role in the pathogenesis of cardiovascular diseases such as hypertension, insulin resistance, metabolic syndrome, and coronary artery disease [9]. However, the role of increased EFT thickness in patients with CSX has not been investigated yet.

In this study, we aimed to investigate the possible role of increased EFT thickness in the pathogenesis of CSX.

*Correspondence to: Macit Kalcik, MD, Department of Cardiology, Hitit University Faculty of Medicine, Çorum, Turkey, E-mail: macitkalcik@yahoo.com

Key words: cardiac syndrome X, coronary artery disease, echocardiography, epicardial fat thickness

Received: October 20, 2018; Accepted: October 29, 2018; Published: October 31,2018 


\section{Methods}

\section{Study population}

Between May 2016 and January 2018, 145 patients (mean age: $54.3 \pm$ 8.2 years , male: 59 ) who diagnosed with CSX and 150 healthy controls (mean age: $55.9 \pm 7.3$, male: 66) were enrolled in this single-center casecontrol study. Patients with left ventricular systolic dysfunction, endstage liver disorders, renal insufficiency, chronic inflammatory diseases, connective tissue diseases and malignancies were excluded from the study. Patients who had angina pectoris with detectable ischemia on noninvasive tests such as treadmill stress test or myocardial perfusion scintigraphy and, without any evidence of stenosis or vasospasm of epicardial coronary arteries during invasive coronary angiography were diagnosed as CSX. Asymptomatic healthy controls without any detectable ischemia on noninvasive tests constituted the control group. All patients were undergoing transthoracic echocardiography (TTE). Complete blood count and blood chemistry panel were carried out in all patients at the time of admission. All demographic, laboratory and echocardiographic parameters were recorded into a dataset and compared between CSX patients and controls. All patients provided a written informed consent and the study protocol was approved by the local ethics committee of the hospital in accordance with the Declaration of Helsinki and Good Clinical Practice guidelines.

\section{Echocardiography}

All patients underwent TTE performed by the same cardiologist using Vivid 5 echocardiography device (GE Vingmed Ultrasound AS, Horten, Norway), and $3.2 \mathrm{mHz}$ adult probe with the patient in the left lateral decubitus position. In all patients, the left ventricular posterior wall thickness (PWT), interventricular septal thickness (IVST), left ventricular end-systolic diameter (LVESD), left ventricular end-diastolic diameter (LVEDD) and left atrial diameter (LAD) were measured on the parasternal long-axis view. Left ventricular ejection fractions (LVEF) of the patients were calculated by using biplane Simpson's method.

\section{Measurement of EFT Thickness}

The measurement of EFT thickness was performed by TTE from a parasternal long-axis view on the right ventricle's free wall at enddiastole and the greatest perpendicular distance to the aortic annulus was achieved and averaged over 3 cardiac cycles [10]. In parasternal long-axis window, hypoechoic space on the of the right ventricular free wall was defined as EFT (Figure 1A).

\section{Statistical analysis}

Statistical analyses were performed using IBM SPSS Statistics for Windows, Version 19.0. (IBM Corp. Armonk, NY). Descriptive statistics are reported as mean \pm standard deviation for continuous variables with normal distribution or median (25th-75th percentiles) values for continuous variables without normal distribution and as frequency with percentages for the categorical variables. The ShapiroWilk and Kolmogorov-Smirnov test was used to test the normality of the distribution of continuous variables. Categorical variables were compared with chi-square or Fisher exact tests. Student $t$ test or MannWhitney $\mathrm{U}$ test was used to compare continuous variables as appropriate. The significance level was accepted as $\mathrm{p}<0.05$ in all statistical analyses. A logistic regression analysis was performed in order to identify any independent associates of CSX. A receiver operating characteristic (ROC) curve analysis was performed to evaluate the sensitivity, specificity, area under the curve (AUC), and confidence interval (CI) of EFT for predicting CSX.

\section{Results}

A total of 145 patients (mean age: $54.3 \pm 8.2$ years, male: 59 ) with the diagnosis of CSX and 150 healthy controls (mean age: 55.9 \pm 7.3 , male: 66 ) were enrolled in this single-center case-control study. Demographic, laboratory and echocardiographic characteristics of the study population are presented in Table 1. Age and gender distribution were similar between patients and controls. There was no significant difference in terms of systolic and diastolic blood pressure measurements and heart rate values between the groups. The prevalences of hypertension, diabetes mellitus, dyslipidemia and smoking status were similar between the groups. Routine serum biomarkers such as glucose, urea, creatinine, uric acid, bilirubin, alanine aminotransferase, aspartate aminotransferase, total cholesterol, high density lipoprotein, low density lipoprotein, triglyceride, and complete blood count parameters were similar between the patients and the controls. The echocardiographic parameters including LVEF, LVESD, LVEDD, LAD, IVST, PWT, left atrial E and A waves were found to be similar between the groups. The mean EFT thickness was significantly higher in CSX patients than the controls $(5.6 \pm 1.1$ vs. $4.9 \pm 0.8 \mathrm{~mm}$; $\mathrm{p}<0.001$ ) (Figure 1B).

The univariate parameters which were possible predictors of CSX were taken into multiple logistic regression analysis. Increased EFT thickness was an independent predictor of CSX (Odds ratio: 1.289;
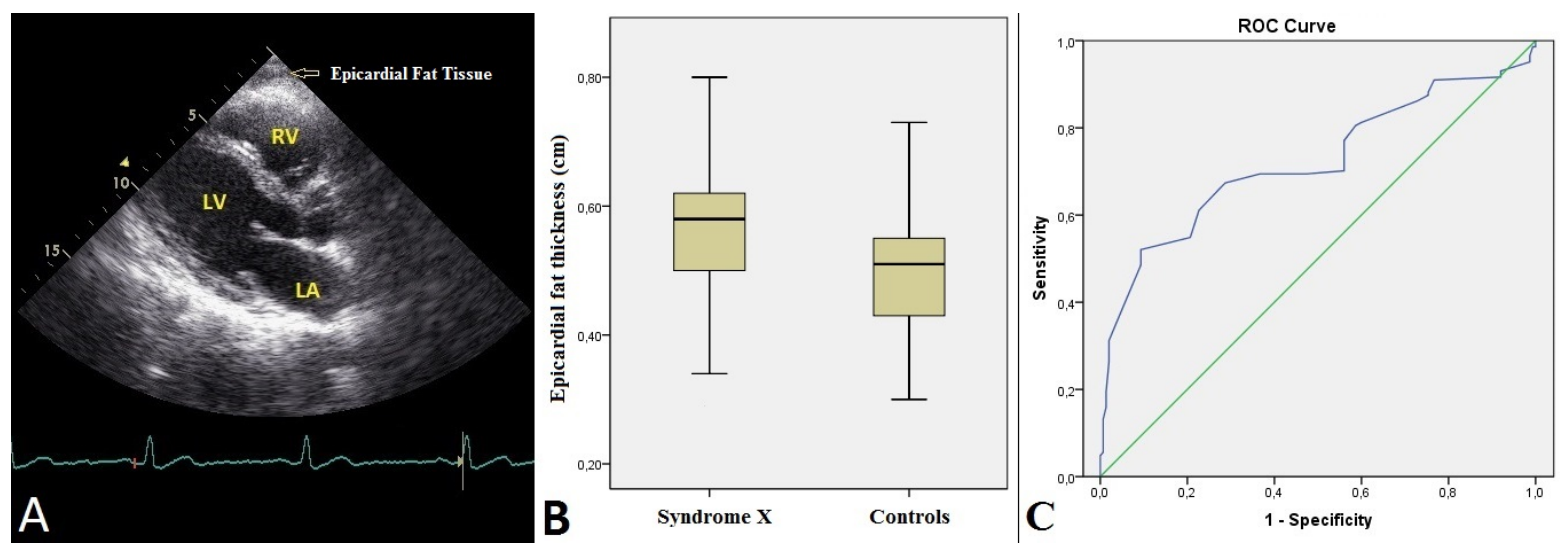

Figure 1. Transthoracic echocardiography revealing the epicardial fat tissue on the right ventricle's free wall from a parasternal long-axis view (A). The box-plot graph comparing the epicardial fat tissue thickness values between patiens with cardiac syndrome $\mathrm{X}$ and the controls (B) Receiver operating characteristic curve revealing the area under the curve for epicardial fat tissue thickness to predict cardiac syndrome X (C). 
Table 1. The comparison of demographic, echocardiographic and laboratory parameters between patient and control groups

\begin{tabular}{|c|c|c|c|}
\hline & $\begin{array}{c}\text { Cardiac Syndrome } X \\
n=145\end{array}$ & $\begin{array}{c}\text { Control Group } \\
\mathbf{n}=150\end{array}$ & P value \\
\hline \multicolumn{4}{|l|}{ Demographic parameters } \\
\hline Age, years & $54.3 \pm 8.2$ & $55.9 \pm 7.3$ & 0.282 \\
\hline Gender-male, $n(\%)$ & $59(\% 40)$ & $66(\% 44)$ & 0.684 \\
\hline BMI, $\left(\mathrm{kg} / \mathrm{m}^{2}\right)$ & $30.1(25.9-33.12)$ & $29.7(26.75-32.4)$ & 0.596 \\
\hline SBP (mmHg) & $137.9 \pm 16.6$ & $134.3 \pm 13.5$ & 0.452 \\
\hline DBP (mmHg) & $83.3 \pm 9.1$ & $82.1 \pm 10.2$ & 0.695 \\
\hline Heart Rate (beats/min) & $78.9 \pm 12.5$ & $74.2 \pm 10.1$ & 0.093 \\
\hline Hypertension, $\mathrm{n}(\%)$ & $49(34)$ & $39(26)$ & 0.133 \\
\hline Diabetes Mellitus, $\mathrm{n}(\%)$ & $24(16.7)$ & $18(12)$ & 0.253 \\
\hline Dyslipidemia, $n(\%)$ & $27(18.8)$ & $37(24.7)$ & 0.219 \\
\hline Smoking status, n(\%) & $33(22.9)$ & $28(18.7)$ & 0.369 \\
\hline \multicolumn{4}{|c|}{ Echocardiographic parameters } \\
\hline LV EF (\%) & $64.9 \pm 5.4$ & $65.7 \pm 4.8$ & 0.452 \\
\hline $\mathrm{LAD},(\mathrm{mm})$ & $38(36-40)$ & $38(36.75-40)$ & 0.584 \\
\hline LVEDD, $(\mathrm{cm})$ & $4.5(4.2-4.9)$ & $4.3(4.1-4.8)$ & 0.275 \\
\hline LVESD, (cm) & $2.98 \pm 0.68$ & $2.92 \pm 0.48$ & 0.617 \\
\hline IVST, (mm) & $11(11-12)$ & $11(11-12)$ & 0.172 \\
\hline PWT, (mm) & $11(11-12)$ & $11(11-12)$ & 0.770 \\
\hline $\mathrm{E}(\mathrm{m} / \mathrm{s})$ & $0.6(0.5-0.7)$ & $0.7(0.5-0.9)$ & 0.372 \\
\hline $\mathrm{A}(\mathrm{m} / \mathrm{s})$ & $1(0.8-1)$ & $0.95(0.7-1)$ & 0.253 \\
\hline \multicolumn{4}{|l|}{ Laboratory parameters } \\
\hline Glucose, $(\mathrm{mg} / \mathrm{dL})$ & $95.3 \pm 11.4$ & $96.7 \pm 10.1$ & 0.882 \\
\hline BUN, (g/dL) & $27.6 \pm 9.6$ & $33.4 \pm 12.3$ & 0.244 \\
\hline Creatinine, $(\mathrm{g} / \mathrm{dL})$ & $0.82 \pm 0.18$ & $0.78 \pm 0.19$ & 0.293 \\
\hline AST, (U/L) & $21(17.75-26)$ & $20.5(18-23.75)$ & 0.521 \\
\hline ALT, (U/L) & $21(13.75-29.25)$ & $21(16-29.5)$ & 0.967 \\
\hline Uric Acid (mg/dL) & $4.91 \pm 1.0$ & $4.80 \pm 1.0$ & 0.754 \\
\hline Total Bilirubin (mg/dL) & $0.6(0.4-0.9)$ & $0.7(0.5-0.8)$ & 0.697 \\
\hline LDL (mg/dL) & $126.75 \pm 27.8$ & $136.8 \pm 38.2$ & 0.294 \\
\hline $\mathrm{HDL}(\mathrm{mg} / \mathrm{dL})$ & $42(35-48)$ & $44(36-51)$ & 0.274 \\
\hline Triglycerides $(\mathrm{mg} / \mathrm{dL})$ & $168.7 \pm 93.8$ & $170.1 \pm 94.7$ & 0.955 \\
\hline Total Cholesterol (mg/dL) & $187.4 \pm 28.9$ & $204.2 \pm 45.2$ & 0.160 \\
\hline Hemoglobin $(\mathrm{g} / \mathrm{dL})$ & $14.17 \pm 1.61$ & $14.03 \pm 1.87$ & 0.869 \\
\hline White Blood Cell, $\left(\times 10^{3} / \mathrm{dL}\right)$ & $7.87 \pm 1.95$ & $8.27 \pm 1.93$ & 0.293 \\
\hline Platelet, $\left(\mathrm{x} 10^{3} / \mathrm{dL}\right)$ & $240.7 \pm 49.7$ & $243.4 \pm 53.2$ & 0.851 \\
\hline
\end{tabular}

(A: Atrial A wave, ALT: Alanine Aminotransferase, AST: Aspartate Aminotransferase, BMI: Body mass index, BUN: Blood urea nitrogen, DBP: Diastolic blood pressure, E: Atrial E wave, HDL: High density lipoprotein, IVST: Interventricular septal thickness, LAD: Left atrial diameter, LDL: Low density lipoprotein, LVEF: Left ventricular ejection fraction, LVESD: Left ventricular end systolic diameter, LVEDD: Left ventricular end diastolic diameter, PWT: Posterior wall thickness, SBP: Systolic blood pressure)

CI\%95: 0.219-0.946; $\mathrm{p}=0.021$ ) (Table 2). In the ROC curve analysis, EFT thickness above $5.3 \mathrm{~mm}$ predicted CSX with a sentivity of $69 \%$ and a specificity of $64 \%$ (AUC: $0.721 ; 95 \%$ CI: 0.661 to 0.781 ; $<<0.001$ ) (Figure 1C).

\section{Discussion}

In this observational case-control study, we have focused on the role of increased EFT thickness in patients with CSX. EFT thickness was observed significantly higher in CSX patients as compared to healthy controls supporting that increased EFT thickness may be associated with mechanisms which play role in the pathogenesis of CSX.

Epicardial coronary artery stenosis is usually responsible for myocardial ischaemia. However, with the technical advancements in the past 30 years, studies have shown that abnormalities in coronary microcirculation may also cause myocardial ischaemia in patients with normal coronary arteries [11]. In 1973, Kemp first described these patients as having CSX, defined as typical angina pectoris with abnormal stress test results indicative of myocardial ischaemia and normal coronary arteries on coronary angiography [12]. Since the original description of syndrome $\mathrm{X}$ over 30 years ago, a large
Table 2. Multivariate regression analysis showing independent predictors of CSX

\begin{tabular}{|l|c|c|c|}
\hline \multicolumn{1}{|c|}{ OR } & $\mathbf{9 5 \%}$ CI & P value \\
\hline Age & 0.993 & $0.961-1.026$ & 0.679 \\
\hline Gender, male & 0.252 & $0.109-0.582$ & 0.092 \\
\hline Hypertension & 0.683 & $0.956-1.038$ & 0.688 \\
\hline Diabetes Mellitus & 0.229 & $0.047-1.121$ & 0.169 \\
\hline Dyslipidemia & 0.772 & $0.256-1.438$ & 0.807 \\
\hline Smoking & 0.975 & $0.482-1.176$ & 0.126 \\
\hline Increased EFT thickness & 1.289 & $0.214-0.946$ & $\mathbf{0 . 0 2 1}$ \\
\hline
\end{tabular}

CI: Confidence Interval; CSX: Cardiac Syndrome X, EFT: Epicardial fat tissue, OR: Odds ratio

number of studies have focused on establishing an ischemic origin for this condition. The pathophysiology of CSX is multifactorial and endothelial dysfunction with subsequent microvascular ischemia has been implicated as an important contributing factor [4].

EFT located beneath the visceral pericardium, is a particular variety of visceral fat tissue. The physiological, biochemical, and biomolecular properties of EFT and the possible paracrine reactions have been reported in previous studies [13]. Various studies have highlighted the potential importance of EFT in relation to inflammatory burden in 
cardiovascular diseases such as coronary artery disease [14], subclinical atherosclerosis [15], and metabolic syndrome [10] and seems to have high capacity of local proinflammatory activity [16]. There is growing evidence that the changes in perivascular tissues surrounding epicardial coronary arteries could alter vascular homeostasis and contribute to endothelial dysfunction, amplification of vascular inflammation, intimal lesions, plaque progression by an outside-to-inside signaling mechanism $[17,18]$.

EFT is present over the base of the heart, the atrioventricular groove, bases of great vessels, along the distribution of coronary arteries, over the RV especially over the free wall and at the apex. Coronary arteries and their epicardial branches are embedded in the EFT [19]. Under physiological conditions EFT functions in the maintenance of fatty acid homeostasis for the local vascular bed and has higher lipogenetic and lipolytic activity than other fat tissues [20]. At the same time EFT is a well-known source of adipokines including a wide range of inflammatory mediators to a larger extent than the subcutaneous and omental fat [21] and secretes vasoactive products that regulate coronary arterial tone [22]. It has also been shown that periadventitial application of inflammatory mediators induces inflammatory cell influx into the arterial wall, coronary vasospasm and intimal lesions [18]. Thus, inflammatory mediators secreted by the EFT surrounding coronary arteries may lead to impaired coronary blood flow reserve through an outside-to-inside cellular cross-talk. Lack of any fascia between the adipocytes and myocardium allows these direct interactions of vascular bed and the surrounding EFT [13]. Supported by the previous studies, increased EFT thickness may be associated with local and paracrine mechanisms that play role in the pathogenesis of CSX.

\section{Study limitations}

The primary limitation was that our study was a nonrandomized and single center study with a relatively small number of patients. Secondly, coronary angiography was not performed in the control group, this issue is also open to criticism. A control group without symptoms and with a negative stress and angiographically normal coronary arteries would have been ideal. However, it would be impossible to get approval from the ethics committee to perform coronary angiography in a patient group without symptoms and with a negative stress test in our country. Lastly, magnetic resonance imaging (MRI) which was known as the gold standard for the measurement of EFT was not used in this study. However widespread use of MRI is not practical for a screening parameter, and echocardiography has been validated against MRI for quantitative assessment of EFT [10].

\section{Conclusion}

EFT thickness values were observed significantly higher in CSX patients as compared to healthy controls. Increased EFT thickness may be associated with mechanisms that play role in the pathogenesis of CSX. EFT has the potential to be an additional and easy diagnostic tool for risk stratification of patients with typical chest pain. Those with an increased EFT are particularly at risk for impaired coronary blood flow reserve which is an early manifestation of atherosclerosis.

\section{Competing interests}

All of the authors have no conflict of interest.

\section{Funding}

The author(s) received no financial support for the research, authorship, and/or publication of this article.

\section{References}

1. Melikian N, De Bruyne B, Fearon WF, MacCarthy PA (2008) The pathophysiology and clinical course of the normal coronary angina syndrome (cardiac syndrome X). Prog Cardiovasc Dis 50: 294-310. [Crossref]

2. Phan A, Shufelt C, Merz CN (2009) Persistent chest pain and no obstructive coronary artery disease. JAMA 301: 1468-1474. [Crossref]

3. Singh M, Singh S, Arora R, Khosla S (2010) Cardiac syndrome X: current concepts. Int J Cardiol 142: 113-119.

4. Hurst T, Olson TH, Olson LE, Appleton CP (2006) Cardiac syndrome X and endothelial dysfunction: new concepts in prognosis and treatment. Am JMed 119: 560-566. [Crossref]

5. Cannon RO III (2009) Microvascular angina and the continuing dilemma of chest pain with normal coronary angiograms. J Am Coll Cardiol 54: 877-885. [Crossref]

6. Chen C, Wei J, AlBadri A, Zarrini P, Bairey Merz CN (2016) Coronary Microvascular Dysfunction- Epidemiology, Pathogenesis, Prognosis, Diagnosis, Risk Factors and Therapy. Circ J 81: 3-11. [Crossref]

7. Schejbal V (1989) Epicardial fatty tissue of the right ventricle morphology, morphometry and functional significance. Pneumologie 43: 490-499. [Crossref]

8. Iozzo P (2011) Myocardial, perivascular, and epicardial fat. Diabetes Care 34 S371-S379. [Crossref]

9. Iacobellis G, Willens HJ (2009) Echocardiographic epicardial fat: a review of research and clinical applications. J Am Soc Echocardiogr 22: 1311-1319. [Crossref]

10. Iacobellis G, Ribaudo MC, Assael F, Vecci E, Tiberti C, et al. (2003) Echocardiographic epicardial adipose tissue is related to anthropometric and clinical parameters of metabolic syndrome: a new indicator of cardiovascular risk. J Clin Endocrinol Metab 88: 5163-5168. [Crossref]

11. Lanza GA, Crea F (2010) Primary coronary microvascular dysfunction clinical presentation, pathophysiology, and management. Circulation 121: 2317-2325. [Crossref]

12. Kemp HG Jr (1973) Left ventricular function in patients with the anginal syndrome and normal coronary arteriograms. Am J Cardiol 32: 375-376. [Crossref]

13. Iacobellis G, Corradi D, Sharma AM (2005) Epicardial adipose tissue: anatomic, biomolecular and clinical relationships with the heart. Nat Clin Pract Cardiovasc Med 2: 536-543. [Crossref]

14. Rosito GA, Massaro JM, Hoffmann U, Ruberg FL, Mahabadi AA, et al. (2008) Pericardial fat, visceral abdominal fat, cardiovascular disease risk factors, and vascular calcification in a community-based sample: the Framingham Heart Study. Circulation 117: 605-613. [Crossref]

15. Iacobellis G, Pellicelli AM, Sharma AM, Grisorio B, Barbarini G, et al. (2007) Relation of subepicardial adipose tissue to carotid intima-media thickness in patients with human immunodeficiency virus. Am J Cardiol 99: 1470-1472. [Crossref]

16. Mazurek T, Zhang L, Zalewski A, Mannion JD, Diehl JT, et al. (2003) Human epicardial adipose tissue is a source of inflammatory mediators. Circulation 108: 24602466. [Crossref]

17. Miyata K, Shimokawa H, Kandabashi T, Higo T, Morishige K, et al. (2000) Rho-kinase is involved in macrophage-mediated formation of coronary vascular lesions in pigs in vivo. Arterioscler Thromb Vasc Biol 20: 2351-2358. [Crossref]

18. Shimokawa H, Ito A, Fukumoto Y, Kadokami T, Nakaike R, et al. (1996) Chronic treatment with interleukin-1 beta induces coronary intimal lesions and vasospastic responses in pigs in vivo. The role of platelet-derived growth factor. J Clin Invest 97: 769-776. [Crossref]

19. Rabkin SW (2007) Epicardial fat: properties, function and relationship to obesity. Obes $\operatorname{Rev} 8$ : 253-261. [Crossref]

20. Marchington JM, Mattacks CA, Pond CM (1989) Adipose tissue in the mammalian heart and pericardium: structure, foetal development and biochemical properties. Comp Biochem Physiol B 94: 225-232. [Crossref]

21. Engeli S, Schling P, Gorzelniak K, Boschmann M, Janke J, et al. (2003) The adiposetissue renin-angiotensinaldosterone system: role in the metabolic syndrome? Int $J$ Biochem Cell Biol 35: 807-825. [Crossref]

22. Sacks HS, Fain JN (2007) Human epicardial adipose tissue: a review. Am Heart J 153 907-917. [Crossref]

Copyright: (C2018 Kalçık M. This is an open-access article distributed under the terms of the Creative Commons Attribution License, which permits unrestricted use, distribution, and reproduction in any medium, provided the original author and source are credited. 